\title{
A Hybrid Time-Domain \\ Maxwell/MTLN-Equations Method to \\ Simulate EM-induced-Currents on \\ Electric Cable-Bundles Inside Cavities
}

\author{
J-P. Parmantier ${ }^{1}$, X. Ferrieres ${ }^{2}$ and P. Schickele ${ }^{1}$ \\ ${ }^{1}$ ONERA/DEMR, Université de Toulouse, F-31055 Toulouse - France \\ ${ }^{2}$ ONERA/DEMR-LMA2S, Université de Toulouse, F-31055 Toulouse - France
}

\begin{abstract}
This paper proposes a time-domain hybrid method for coupling MulticonductorTransmission-Line Network equations and a Finite Element Method to evaluate the electromagnetic response of the electric wires of a cable-bundle located inside a 3 dimensional structure. The method is applied and demonstrated over a box structure made of several volumes containing a realistic multiconductor cable-harness and illuminated by a plane wave. The formalism of the method is given and the results obtained show the interest of this approach.
\end{abstract}

INDEX TERMS Maxwell-equations, Finite Element Method (FEM), Multiconductor-Transmission-Line Network (MTLN), Time-domain hybridization.

\section{INTRODUCTION}

We are interested in the electromagnetic (EM) response of a system composed of an electrical wiring located inside a 3D structure. Considering the characteristic dimensions of the three-dimensional (3D) structure and the bundles of electric wires, such a problem is a typical multiscale problem for which both components require specific equations that must be coupled between each other in order to be able to handle all the relevant parameters of the entire EM problem.

On the one hand, 3D numerical methods solving Maxwell's equations are now very mature to describe EM scattering by and into realistic structures. Many techniques exist with their advantages and their drawbacks depending on the type of problem to address [1][2][3][4]. However, including wire network models into 3D EM numerical techniques is always a challenge. We may mention common issues related to the description of wire routes in discretized 3Dgeometrical models or issues for maintaining the stability of the global numerical scheme. By the way, many wire models [5][6][7][8] embedded in 3D schemes cannot handle the specificities of multiconductor networks : large number of wires in branches, large variety of cable types, complex connections at junctions and various types of load terminations.

On the other hand, Multiconductor Transmission-Line Network (MTLN) models allow capturing all those specificities [9]. However they are limited by the Transmission-Line (TL) theory which requires the quasi Transverse Electromagnetic
(TEM) approximation. Of course, nowadays, very efficient hybridization techniques based on Field-to-TransmissionLine (FTL) [10] allow users to couple MTLN equations together with Maxwell's equations. Nevertheless, they are still limited by this TEM approximation which makes the application of FTL impossible at high frequencies or when a reference return conductor cannot be clearly defined for the TEM model.

In this paper, we investigate a hybrid numerical method that links TL-equations together with the Maxwell-equations and that overcomes the previously mentioned limitations. For this purpose, the capability to link both EM fields and TL-induced currents including the reaction of one on the other becomes a requirement. To this extent, we think of applying a hybridization technique entirely set in the time-domain making possible this reciprocal EM-field-toTL-currents reaction applicable at each time step of the calculation. However, we want this hybridization technique flexible enough in order to be able to choose the most appropriate Maxwell-equation numerical scheme for the calculation of the EM fields. In addition, we want to handle excitation sources generated either as EM fields in the 3D Maxwell calculation domain or voltage/current generators on the MTLN calculation domain.

In order to validate our method and demonstrate its flexibility we propose to apply it on a specific system made of an electrical conductive box containing several volumes in which a complex wiring harness is installed and illumi- 
nated by an incident plane wave. Due to the rectangular geometry of the 3D structure we want to show how a dedicated FEM method may improve the performance and the precision of the field calculation. In section II of this paper, we describe the application problem that will lead our hybridization technique development and validation. In section III, we introduce the formalism of our time-domain hybridization technique and we put it into perspective with the well-known thin-wire model. In section IV, we apply this technique on our application test-case. First we show the validation of our Multiconductor-Transmission-Line (MTL) equation numerical scheme and introduce a FEM technique dedicated to a cartesian meshed model of the geometrical problem. Then we show the validation of the hybridization process and how a MTLN model to account for topological specificities of wiring harnesses is required to overcome the thin-wire model limitations. Finally, we conclude on the achievements of this paper and introduce prospects for future improvements of our hybridization technique.

\section{PHYSICAL PROBLEM DRIVING THE DEVELOPMENTS}

The structure leading our development is a parallelepiped box called NTC2, as "Numerical Test-case 2", that has been defined and studied in a former H2020 European Project called EPICEA, 2017-2019, (grant agreement $n^{\circ} 689007$ ) [11]. It consists of 3 interconnected cavities (Cavity 1, 2 and 3), $100 \mathrm{~cm} \times 90 \mathrm{~cm} \times 70 \mathrm{~cm}$ each, in which an electrical wiring is installed. The various dimensions are given in Figure 1 with respect to a $x, y, z$ reference system. In the Figure, red and blue strips display current return networks that do not have to be considered in our problem since they are melted with the PEC faces and walls. The exterior faces of the box are labelled $T i, i=1,3, D i, i=1,3$, $R i, i=1,3, L i, i=1,3$ with end-faces noted $\mathrm{F} 3$ and $\mathrm{B} 1$. The separation walls between the 3 cavities are labelled S1-2 and S2-3. The box is provided with 3 openings to the outside labelled A1, A2 and A3, which are points of penetration and loss of EM energy from the outside and to the outside. The separation wall S1-2 is also provided with an internal aperture labelled A1-2 allowing energy flowing between the two separated cavities. In our problem, all the materials have been considered PEC (Perfectly Electrical Conductors).

A wiring is routed into the 3 cavities, along 3 branches connecting B1, R2 and F3 faces, going through the separation walls S1-2 and S2-3 into small holes called H1-2 and H2-3. The topology of the 3 branches is as follows :

- from the R1 face, Branch 1, 1.95m-long, runs along the $\mathrm{z}$ axis in Cavity 1, in parallel to the corner made by L1 and R1 faces, crosses wall S1-2 into Cavity 2, in parallel to the corner made by faces L2 and R2, and ends up at a separation junction, just before the small hole H1-2 ;

- Branch 2, 0.55m-long, runs along the $\mathrm{z}$ axis from this separation junction into Cavity 3 , in parallel to the corner made by faces L3 and R3 though the small hole $\mathrm{H} 2-3$;
- Branch 3, 0.45m-long, runs along the y axis in Cavity 2, in parallel to the corner made by the S2-3 separation wall and R2 face.

All 3 branches are at $5 \mathrm{~cm}$ from all closest faces and walls. They end-up at terminations labelled Ter1 for Branch 1, Ter 2 for Branch 2 and Ter3 for Branch 3.

Lossy dielectric cubes, $20 \mathrm{~cm} \times 20 \mathrm{~cm} \times 20 \mathrm{~cm}$, are located in each cavity as shown in Figure 1 in order to lower the quality factors, as encountered in real industrial structures. In real structures, such losses are brought by furniture, electric and electronic equipment, passengers, wall and floor coating etc... The electrical properties of each cube are: relative dielectric permittivity $\varepsilon_{r}=2.5$ and electric conductivity $\sigma=3.7 \mathrm{mS} / \mathrm{m}$.

As far as the EM excitation is concerned, the structure is illuminated by an incident plane wave whose characteristics are as follows :

- the wave vector $k$ is located in the $x z$ plane, inclined by $45^{\circ}$ relatively to the $\mathrm{z}$ axis ;

- the electric field, $E$, is also located in the $x z$ plane, inclined by $45^{\circ}$ relatively to the $\mathrm{x}$ axis ;

- the magnetic field, $H$, is along the $\mathrm{y}$ axis.

The time dependent waveform applied to the plane waveexcitation is a Gaussian pulse given by $f(t)=A \exp (-((t-$ $\left.\left.\left.t_{0}\right) / a\right)^{2}\right)$, with $A=1 \mathrm{~V} / \mathrm{m}, t_{0}=7 E-10 \mathrm{~s}$ and $a=2.5 E-$ $10 \mathrm{~s}$.

The electrical wiring topology is described in Figure 2. The wiring is made of several types of electrical wires following branches 1,2, 3 and connecting impedance networks located at termination junctions called J1, J5 and J6. A junction $\mathrm{J} 3$ allows the connection between the electric wires at the level of the junction of the 3 branches. Junctions $\mathrm{J} 2$ and $\mathrm{J} 4$ simply provide the continuity of the electric wires when crossing into H1-2 and H2-3 holes.

In Branch 1 running between junctions $\mathrm{J} 1$ and $\mathrm{J} 3$, the wiring is made of a $50 \Omega$ (characteristic impedance) coaxial cable and a single-wire cable, both of them located inside an overshield. In Branch 2, the coaxial cable and single wire of Branch 1 are continuing their route up to junction $\mathrm{J} 5$ to which a pair-cable is added. The 3 types of cables are inside an overshield with the same electrical characteristics as in Branch 1, thereby prolonging the overshield on Branch 1. In Branch 3, the two wires of the pair-cable of Branch 2 are continuing inside a shield, becoming therefore a shielded pair cable. At the level of junction J3, perfect connection between all the electric wires in each branch and between all cable shields is provided. The single wire in branches 1 and 2 is connected to $10 \mathrm{k} \Omega$ common-mode resistances at both ends. The wire of the coaxial cable is connected to $50 \Omega$ common-mode resistances at both ends. The wires of the pair running in Branch 2 and Branch 3 are connected to two differential loads made of $1 k \Omega$ resistance in series with a $2 n F$ capacitance.

All cable shields, the coaxial-cable shield, the shieldedpair shield and the overshield are connected at their ends, 


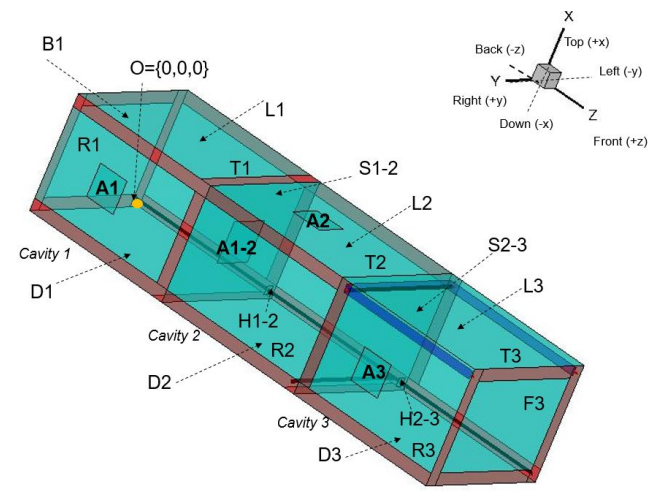

(a) Topological description and wiring routes

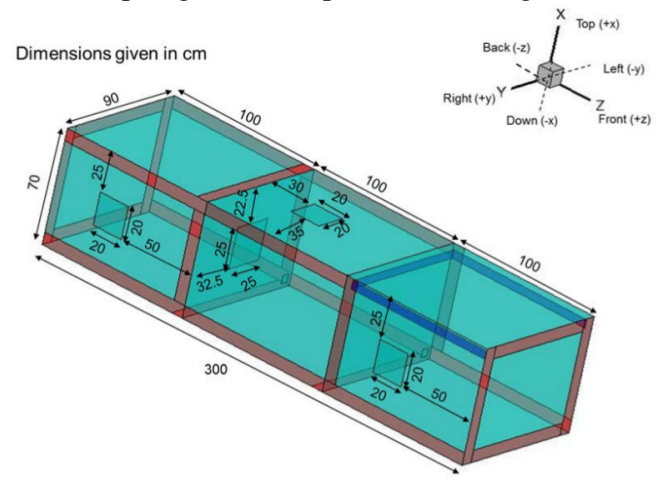

(b) Main dimensions

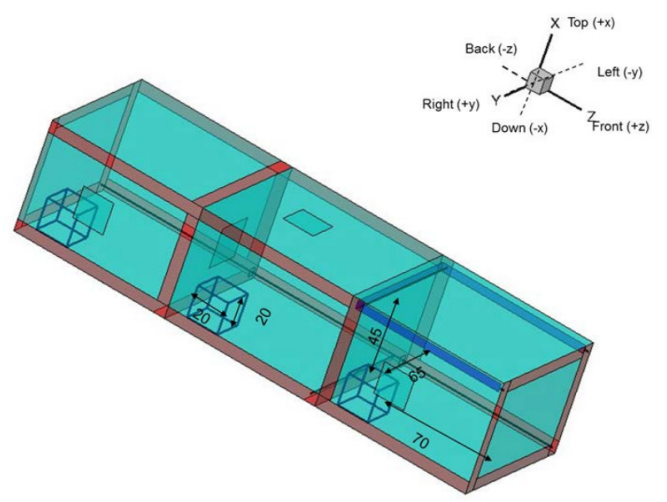

(c) Absorbing cubes, locations and dimensions

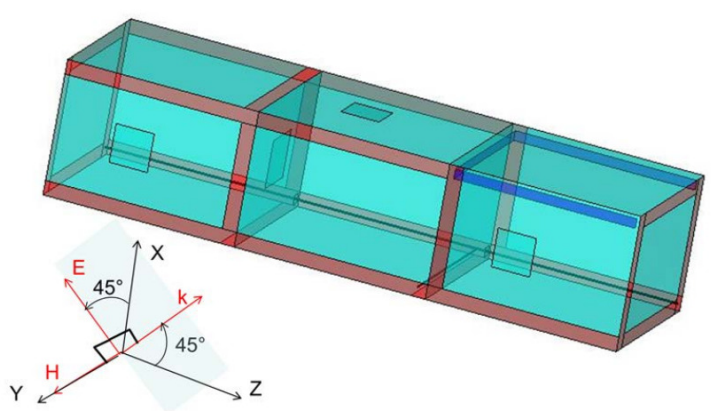

(d) Plane wave excitation

FIGURE 1: 3D-structure problem geometry, absorbing elements, dimensions and source excitation. at the levels of junctions $\mathrm{J} 1, \mathrm{~J} 5$ and $\mathrm{J} 6$, respectively corresponding to the connection points Ter1, Ter2 and Ter3 in the structure. No other connection to the structure is considered along the branches between those 3 junctions. Note that all shields and cable shields are considered as equivalent wires of the multiconductor bundles and labelled as such in Figure 1.

Actually, such a wiring topology has been designed on purpose to show how a shielding strategy may be entirely broken by an inappropriate management of the shield connections. Indeed, the overshield in branches 1 and 2 is mounted properly, with its extremities connected to the structure, which enables maximum current circulation on it. However, the expected shielding effect on the whole network may be totally ineffective for two main reasons. First, the connections of this overshield to the structure should be done when crossing walls at the levels of $\mathrm{J} 2$ and $\mathrm{J} 4$ in order to confine EM field in each of the 3 cavity volumes of the 3D-structure and avoid shield current conduction from one volume to the other. Second, and especially, this overshield topology is not maintained in branch 3 in which the shield of the shielded pair is connected to the shield of the coaxial cables running in branches 1 and 2. If some EM coupling happens in branch 3, the induced current on this shield is likely to separate into the two shields of the coaxial cables and thereby drive some currents inside the overshield, which is not wanted. A proper topology connection would have been to connect the shield of the shielded pair to the overshield at the level of $\mathrm{J} 3$.

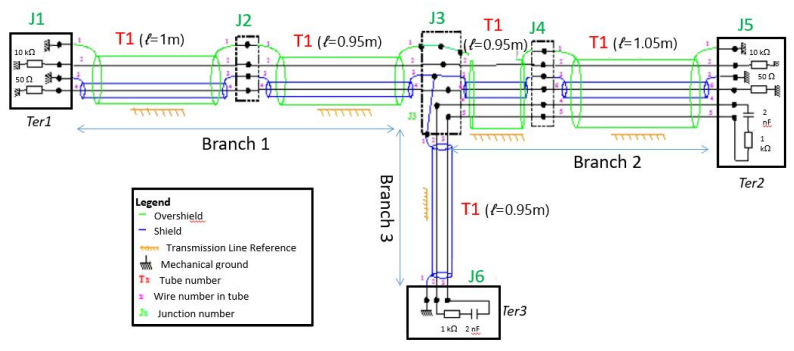

FIGURE 2: Wiring topology under study.

\section{NUMERICAL MODEL PROPOSED TO SIMULATE THE PHYSICAL PROBLEM.}

Two main modelling strategies are available in the literature to assess EM coupling on cables at system level :

- a simplified modeling of the wiring integrated into Maxwell's equations, also known in the literature as "thin-wire" formalism [5] ;

- a FTL hybridization method between a MTLN equation for determination of the wiring electrical response and Maxwell's equations to evaluate the wiring voltage and current equivalent generators coming from incident EM field terms [12]. Among those models, the Agrawal model is very appropriate from a numerical modelling 
point of view because it requires only voltage generators equal to the tangential incident electric fields along the MTLN routes [13].

For a precise modelling of a real wiring problem including various types of cables, in terms of propagation, EM shielding, cross-coupling inside bundles, and topology in terms on connectivity of those cables, FTL is very appropriate [14],[15] and is now commonly accepted for complex wiring modelling at system level [10], [16]. However, its application suffers from a major approximation which is the absence of scattered EM field reaction of the wire induced currents over the incident fields. In the two following paragraphs, we briefly present the mathematical equations of the thin-wire and FTL strategies and the improvements we propose to FTL in order to establish an hybridization technique that solves this field reaction limitation.

\section{A. THIN WIRE FORMALISM IN TIME-DOMAIN VOLUME 3D TECHNIQUES}

The well-known formalism proposed by R. Holland [5] can be written by the system of equations :

$$
\left\{\begin{array}{l}
\varepsilon \partial_{t} E+\sigma E+\mathbf{J}=\nabla \times H \\
\mu \partial_{t} H=-\nabla \times E \\
\partial_{t} I=-\nu^{2} \partial_{l} q-\frac{\mathbf{E}}{L} \\
\partial_{t} q=-\partial_{l} I
\end{array}\right.
$$

where $E, H, I$ and $q$ are respectively the electric and magnetic fields in the computational domain $\Omega \subset \mathbb{R}^{3}$ and the currents and charges on the wire structures, in the computation domain $\Omega_{l} \subset \mathbb{R}$. The terms $\mathbf{E}=E \cdot u$, with $u$ the vector director of the wire, and $\mathbf{J}=I / S$ are coupled terms which are given by the computed electric fields $E$ in $\Omega$ and the electrical currents $I$ evaluated on the wires. $\nu$ defines the propagation velocity of the medium characterized by its permittivity $\varepsilon$ and its conductivity $\sigma . S$ is homogeneous to a surface (see [5]) and the quantity $L$ is, an inductance value given by the thin-wire formalism. The reader will refer to [5] for more details on these definitions.

To complete the system of equations (1), a condition on the electric field is added for perfectly metallic walls, given by $n \times E=0$, where $n$ is the normal to the wall. In addition, PML (Perfectly Matched Layers) [18] are used to bound the computational domain $\Omega$ and the condition $n \times E=0$ is set on the boundary $\partial \Omega$ of this computational domain.

At wire ends, several conditions can be considered :

- $q=0$, the electric charge is null when the wire is connected to a PEC surface ;

- $I=0$, the total current on wire is null when the wire is not connected to any surface or any wire ;

- $q= \pm I / \nu$ defines the electric charge when the wire is assumed to be infinite ;

- for $n$ wires $w_{i}, i=1, n$, connected to each other, we have $\sum_{i=1}^{n} \bar{I}_{i}=0$ and $\forall i=1, n, V_{i}=V_{0}$, where $V_{0}$ is a real value, $V_{i}$ is the voltage at the end of the wire $w_{i}$, and $\bar{I}_{i}$ is the signed current (+ when going into the junction, - when outgoing from the junction). Also for any wire $w_{i}$, the voltage $V_{i}$ at one of its extremities, is also equal to $L_{i} \nu^{2} q_{i}$ (we have $L_{i} C_{i}=1 / \nu^{2}$ and $V_{i}=q_{i} / C_{i}$ ), with $L_{i}$ the inductance of the wire and $q_{i}$ the electric charge at this extremity.

In addition to the fact that each network branch is modelled as a unique wire, the general numerical constraint of this model is the fact that the wires must be considered as thin wires, i.e. their radius are much smaller than the size of the mesh-cell. Doing so, the calculated currents can be seen as "averaged currents" on network branches and it becomes impossible to distinguish the currents in each elementary cable in the branches. Another disadvantage of the Holland model is that the wire directions must follow the mesh cells. Nowadays, several models called "oblique wires" [6], [7], [8] have been proposed in the literature. However, whatever those improvements, the current calculated in the thin-wire models is not equal to the total branch current since the connectivity at junctions and end loads of each wire is not accounted for. This limitation may result in significant errors, especially at low frequency or late time responses.

To mitigate such drawbacks, a model has been proposed in [19] that makes it possible to handle multiconductor cables made of several wires provided that the wires radius (as well as the distance between wires) remain small compared to the mesh-cell size and run within a homogeneous medium in each mesh-cell. Unfortunately, those limitations together with the limited possibilities of connections between several wires at wire-junctions make this problem not applicable for realistic wire system modelling.

\section{B. HYBRID MAXWELL/MTLN EQUATION}

As for the thin-wire formalism, the principle of the hybrid method consists in coupling two systems of equation : one system, based on Maxwell's equations, to evaluate the EM fields and one system, based on MTLN equations, to evaluate the induced currents on the wires of the MTLN model. Unlike for the thin-wire formalism, this hybrid method allows the user to consider complex network topologies made of a large variety of multiconductor cables and wire junctions, including electrical circuit networks at wirejunctions and end-loads. The network model description uses a topological model made of junctions and tubes representing respectively the wiring junctions and branches according to EM topology [20] (see Figure 3). Each tube $k$ supports a MTL model. Figure 4 displays the elementary circuit-cell of a 2 wire MTL labelled $k$. The electrical properties of this circuit are described by series $Z_{k}$ per-unitlength (p.u.1.) impedance and $Y_{k}$ p.u.l. admittance matrices. A usual and convenient approximation consists in decomposing those two matrices in two not-frequency-dependent parts with a resistance $R_{k}$ matrix and a conductance $G_{k}$ matrix respectively and two linear frequency $(\omega=2 \pi f, f$ : frequency) varying parts with a p.u.l. inductance $L_{k}$ matrix and a p.u.l. capacitance $C_{k}$ matrix respectively. All those 
matrices depend on the geometry cross-section and electrical properties of the MTLs [21]. As far as cable-shields and bundle-overshields are concerned they also include the transfer impedance $(Z t)$ properties of those shields [22]. In our approach, this model is based on a common reference conductor in which all the TL currents of the wires return.

In the general MTLN theory, this reference can be made by the nearest structure to the cable or one of the wires in a cable bundle. On the one hand, FTL theory, imposes to set this reference as the closest structure. On the other hand, our hybrid numerical modelling strategy, as in the Holland formalism, makes it possible to consider the set of mesh-cells surrounding the cable bundle as a fictitious and equivalent reference conductor. It appears when deriving the TL or MTL equations in the 3D model but unlike the real electric wires of the electric conductors it does not have real existence. It offers several advantages :

- the identification of the "closest structure" is not always easy to make when this one is not plane. This is for example the case for network branches running along corners or over curved surfaces. Encapsulating the MTL model within surrounding mesh-cells makes them fully independent from the surrounding structure ;

- even if the "closest structure surface" can be identified, its geometry or the height of the network branches with respect to this structure may change, which makes the related MTL models become Non-Uniform Transmission-Lines (NUTL) models. In other terms, the MTL cross-section changes with the position along the tubes. The resolution of NUTL requires approaches that are all equivalent to breakdown the MTL in small uniform MTLs [22], which makes the calculation of course most costly than usual uniform MTLs. Our timedomain formulation of MTL model naturally requires such a decomposition in elementary cells as in Figure 3 and the fact of sampling those MTL cells is not a limitation and allows us to consider NUTL models when, for example, the geometry of the surrounding cells vary along the wires ;

- the TEM approximation must be verified and remains a limitation for the application of MTL models. However, when the reference is taken in the mesh-cell, the dimension of the mesh-cells is generally smaller compared to the height of the bundle to the closest structure surface (again, when this one can be identified), which pushes ahead the frequency limit of the model.

The equations of the model are given by the Maxwellequations and $N$ transmission lines equations corresponding to the $N$ tubes in the network configuration. Let $\Omega \subset \mathbb{R}^{3}$ and $\forall k=1, N, \Omega_{k} \subset \mathbb{R}$, be respectively the computational domains for the EM fields and the $I_{k}$ and $V_{k}$, electrical current and voltage vectors. Then, we search $\left.(E(t, x), H(t, x)) \in \mathbb{R}^{6}, \forall(t, x) \in\right] 0, T[\times \Omega$ and for all $k=1, N,\left(I_{k}(t, \ell), V_{k}(t, \ell)\right) \in \mathbb{R}^{2 m_{k}}$, with $m_{k}$, the number

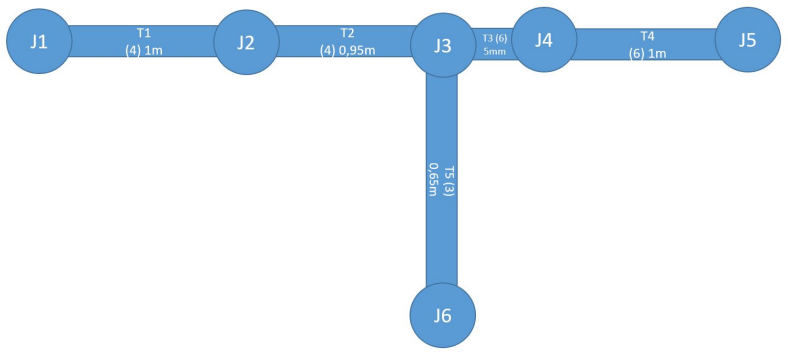

FIGURE 3: Topological model of the Figure 2 wiring network under study. The network model is made of junctions (J1 to J6) and tubes (T1 to T5). "(x)" denotes the number of conductors in the tubes.

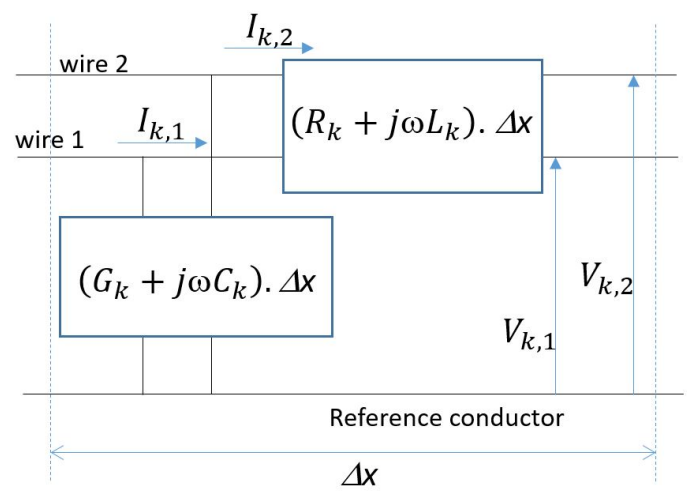

FIGURE 4: Two-wires Multiconductor Transmission Line elementary circuit cell model.

of wires in the cable $k$, and $(t, \ell) \in] 0, T\left[\times \Omega_{k}\right.$, so that :

$$
\left\{\begin{array}{l}
\varepsilon \frac{\partial E}{\partial t}+\sigma E+J=\nabla \times H \\
\mu_{0} \frac{\partial H}{\partial t}=-\nabla \times E \\
E(t=0, .)=H(t=0, .)=0 \quad \text { on } \Omega \\
\forall t \in] 0, T[, n \times E(t, x)=0 \quad \forall x \in \partial \Omega \\
\text { On } \Omega_{k}, \quad \forall k \in\{1, \ldots, H\}: \\
\quad L_{k} \frac{\partial I_{k}}{\partial t}+R_{k} I_{k}=-\frac{\partial V_{k}}{\partial \ell}-E \cdot u_{k} \\
\text { On } \Omega_{k}, \quad \forall k \in\{1, \ldots, N\}: \\
\quad C_{k} \frac{\partial V_{k}}{\partial t}+G_{k} V_{k}=-\frac{\partial I_{k}}{\partial \ell} \\
I_{k}(t=0, .)=V_{k}(t=0, .)=0 \quad \text { on } \Omega_{k}, \forall k \in\{1, \ldots, H\} \\
+ \text { boundaries conditions on } J U_{m}, \forall m \in\{1, \ldots, M\}
\end{array}\right.
$$

where $T$ defines the observed time, $n$, the outward normal at the boundary $\partial \Omega$ of the domain $\Omega, u_{k}$ the director vector of the cable $k$ and $\forall m=1, M, \quad J U_{m}$ is the junction $m$ in the topological network. The number of junctions in the network is equal to $M$. In these equations, $R_{k}, L_{k}, G_{k}$ and $C_{k}$ are 
the matrices of size $m_{k} \times m_{k}$ that define respectively the p.u.l. resistance, inductance, conductance and capacitance matrices as described in Figure 4.

As for the thin-wire formalism, the two coupling terms are defined by the current density $J$ and the tangential electric field component along the wire path, $E \cdot u_{k}$. Like in the thin-wire formalism, we indeed define an averaged path (also called "central path") for each tube on which we compute the electrical fields required to evaluate the $E \cdot u_{k}$ terms [13]. Next, this value is injected as a voltage generator source term distributed along the length of each wire of the tube $k$. Then, we can evaluate the current vector, $I_{k}$, whose components are the currents on all wires of the tubes. The sums of those currents on each tube $k, I_{t o t, k}$, act themselves as distributed current density sources $J_{k}$ that generate an EM scattered field correction to obtain the total field. These sources are defined as $J_{k}=I_{t o t, k} / S$, where $S$ is equivalent to a surface (section of the cable bundle modelled by the tube $k$ ) (see [23][24]). This model is valid as far as the MTL models cables grouped together in bundles for which the applied tangential electric field, $E \cdot u_{k}$, can be supposed to be indistinctly the same for all the wires and for which the distributed current density source $J_{k}$ can be supposed as supported by the central path of the bundle. This model also supposes that, in our modelling approach, there will be only one central path in each cell of the 3D model. This approach also allows the user to account for EM coupling between cable bundles running in close central paths. The EM coupling between cable bundles is made by the exchange of EM fields in the Maxwell-equations.

\section{IMPROVEMENTS OF THE HYBRID MTLN/MAXWELL'S EQUATIONS}

As said before, the FTL is a simplified form of a Maxwell's equations/MTLN hybridization strategy. In most references, the FTL is applied in the frequency-domain because of the possibility offered to the user to run separately the EM field calculation and the MTLN calculation. The numerical interest of this weak hybridization is that the MTLN calculation is much faster than the Maxwell calculation. In particular, the EM field source term calculation to be applied in the MTLN model can be done once for all and several MTLN models can be run for those same EM source terms, provided that the central path of the MTLN model is not changed. This is why a frequency-domain MTLN equation such as the Baum Liu Tesche (BLT) equation of EM topology is particularly appropriate. When timedomain solvers are used for the EM field calculation, those fields have to be Fourier transformed. This is the case for example for references [14], [15] and [17] for which the Yee-scheme [25] is used to deal with Maxwell's equations. Even if this approach is valid at low frequency (under the upper validation limit of the TEM mode), the absence of scattered EM fields generated by the wire induced currents remains a simplification that limits the validity scope of the approach, especially at high frequencies when cables are strongly EM-coupled with their 3D environment, as for radiating antennas.

Let us also stress the point that Agrawal's FTL approach is not a simple simplification of our time-domain hybridization technique that could be obtained by neglecting the $J$ source term in the first equation of (2). Indeed, we remind that the FTL hybridization formulation requires MTL models referenced to the structure [12] whereas our hybridization technique requires MTL models referenced to cells surrounding the MTL central path. The counterpart of our approach is that the EM field calculation and MTLN calculation cannot be performed separately as for the FTL approach applied in the frequency-domain.

In our hybridization approach, the problems associated with signal processing are thereby avoided by using an entire system of equations in the time-domain. The resolution of the MTL-equations is made by decomposing each MTL into $N s$ elementary cells (segments) as in Figure 4 for which the elementary length $\Delta x$ is equivalent to an averaged value of the cell-size in the 3D mesh. The coupling term $E \cdot u$ on each segment is calculated as an average of the $E_{k_{i}}$ terms, which requires splitting each segment $S_{i}$ into $n_{i}$ parts so that each part is inside a cell. Then, for the segment $S_{i}$ :

$$
E . u=\frac{\sum_{j=1, n_{i}} \int_{l_{j} \in \text { Supp }_{S_{i}}} E_{K_{j}} \cdot u d l}{\sum_{j=1, n_{i}} \int_{l_{j} \in \text { Supp }_{S_{i}}} d l}
$$

where $\operatorname{Supp}_{S_{i}}$ is the set of cells $K_{j}$ with a non-zero intersection with the segment and $l_{j}$ the length-part of the segment inside the cell $K_{j}$.

Then, on each wire of the segment, the electrical current is evaluated by using a finite difference leap-frog scheme in time and in space [5], except at the extremities of the wires belonging to junctions. At this level, the currents on wires are evaluated by solving a linear system corresponding to the electric circuit network linking those currents to the voltages developed in the junction circuit components and at connection points. For processing the practical problem presented in section II, in addition to ideal junction models such as short-circuits, open circuits and ideal connection between wires (without losses), we have thereby considered end-circuit networks with electrical circuit components as shown in Figure 5.

The fact of defining a TL model with respect to a reference conductor may question the way to manage connections of the wires at their ends as well as, from a general point of view, the connections between wires. In our modelling approach, all the loads are lumped loads compared to the geometrical dimensions of the problem. Thereby they do not have any geometrical dimension. They will always be connected to the 3D structure or to electrical wires on which it will be always possible to apply our TL model. Some issues can be raised for TL resistance losses that may not be considered as lumped. In such a case, we will distribute the total resistance of interest into a wire model. 


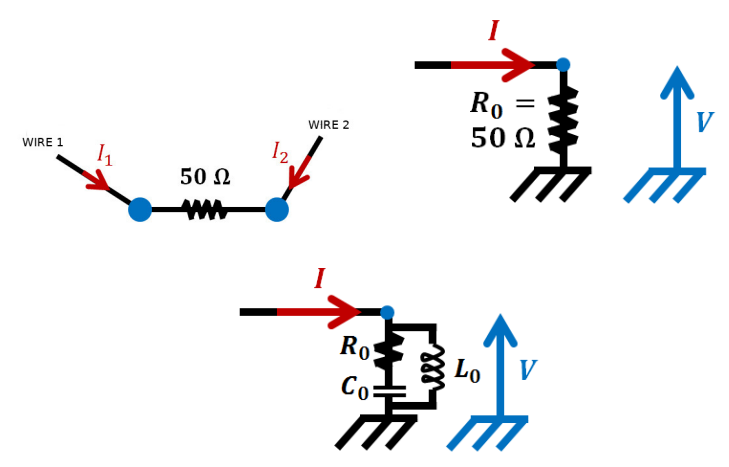

FIGURE 5: Junction circuit models used in the Figure 1 multiple cavity test-case. The figure displays the voltages and currents to be linked to the MTLN voltages and currents.

\section{APPLICATION ON THE RECTANGULAR CAVITY TEST-CASE}

In this section, we apply our hybrid method to solve the problem presented in section II. Here, we want to show the interest of our hybridization method to address the wiring network installed in the rectangular-shape object. In particular, we want to show the advantage of combining both an accurate Maxwell-equation scheme for evaluating the required electric fields as sources of the MTLN model and a method accurate enough to describe correctly any wiring harness topology.

As a first step, we want to validate our time-domain numerical scheme. First, we compare our MTLN technique to a reference frequency-domain MTLN scheme for a selfsufficient configuration of the Figure 2 wiring. Then we describe the formalism of a specific Finite Element Method (FEM) scheme to be applied on a cartesian mesh of course well adapted to our rectangular object.

As a second step, we investigate the validation of the hybridization process between the two time-domain numerical schemes. At the beginning, we focus on the exchange of field and current source terms in our hybridization process on a simplified configuration called "single-wire network".

Then, we show the need to describe correctly the topology of a wiring network. To this extend, we will consider the real "multiconductor network" problem of section II, with all the cables and the real connections between the electric wires. In order to show the limitation of the usual thin-wire model, we will compare the total branch currents of this real network configuration with the currents on the wires in the single-wire network configuration.

In this section, all the results are presented in the timedomain in order to highlight the advantage of this method as far as time-domain results are the objective of the analysis.

\section{A. VALIDATION OF OUR MTLN NUMERICAL SCHEME}

Figure 6 shows some comparison results in the frequencydomain between our MTLN formalism (Fourier transformed results) and a reference BLT formalism-based reference solver working in the frequency-domain, CRIPTE [26]. The excitation of the wiring is such that there is no need of Maxwell's equation to obtain the wiring electrical response; for this purpose, a voltage generator is applied on the first conductor of tube T1 at the level of junction J1 (refer to Figure 2). In the two methods, MTL p.u.l. parameters are chosen as the closest reference structure. In the first comparison (a), the wire extremities are all loaded on $50 \Omega$ at junctions J1, J5 and J6. In the second comparison (b), the real circuit components and real circuit junctions are considered as described in Figure 2. The frequency band of analysis has been chosen low enough such that the TEM mode approximation remains acceptable.

The results in Figure 6 are similar in the two methods and provide a good validation of our MTLN solver. The small differences observed on the resonance peaks are due to the difference of frequency sampling made in the frequencydomain calculation and imposed by our Fast Fourier Transform process.

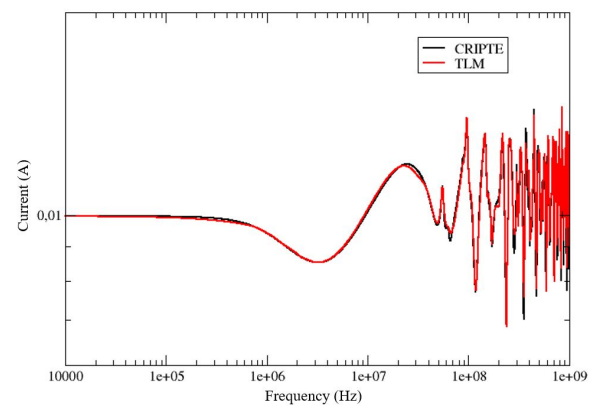

(a)

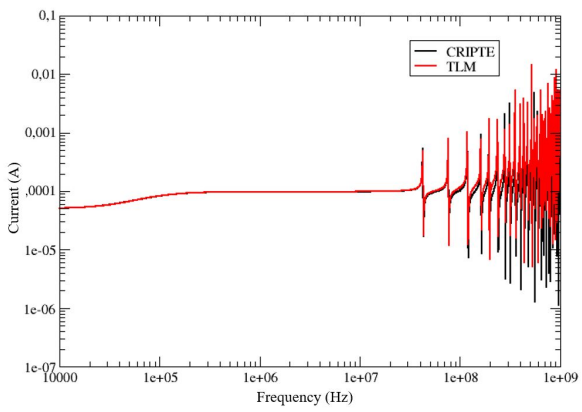

(b)

FIGURE 6: Current observed on wire 2 of tube T1 for the wiring network of Figure 2.

(a) : all end loads equal to $50 \Omega$. (b) : real loads.

Comparison between a reference BLT solver (named "CRIPTE") and our time-domain MTLN solver (named "TLM").

Excitation: voltage generator on wire 1 of tube $\mathrm{T} 1$ at the level of junction $\mathrm{J} 1$. 


\section{B. DEDICATED FEM MODEL FOR THE MAXWELL-EQUATION RESOLUTION}

As far as the evaluation of EM fields is concerned and considering the parallelepiped shape of the 3D structure, we introduce a high-order spatial approximation FEM applied on a cartesian mesh. This method allows us to compute an accurate solution by reducing numerical dispersive errors compared to usual 3D methods [8], [27]. The numerical approximation of electric and magnetic fields on a cell $K$ are given by :

$$
\begin{aligned}
\vec{E}_{K}(t, x, y, z)= & \sum_{l_{1}=1}^{r} \sum_{l_{2}=1}^{r+1} \sum_{l_{3}=1}^{r+1} E_{K, l_{1} l_{2} l_{3}}^{x}(t) \vec{\varphi}_{l_{1} l_{2} l_{3}}^{x}(x, y, z)+ \\
& \sum_{l_{1}=1}^{r+1} \sum_{l_{2}=1}^{r} \sum_{l_{3}=1}^{r+1} E_{K, l_{1} l_{2} l_{3}}^{y}(t) \vec{\varphi}_{l_{1} l_{2} l_{3}}^{y}(x, y, z)+ \\
& \sum_{l_{1}=1}^{r+1} \sum_{l_{2}=1}^{r+1} \sum_{l_{3}=1}^{r} E_{K, l_{1} l_{2} l_{3}}^{z}(t) \vec{\varphi}_{l_{1} l_{2} l_{3}}^{z}(x, y, z) \\
\vec{H}_{K}(t, x, y, z)= & \sum_{l_{1}=1}^{r+1} \sum_{l_{2}=1}^{r} \sum_{l_{3}=1}^{r} H_{K, l_{1} l_{2} l_{3}}^{x}(t) \vec{\psi}_{l_{1} l_{2} l_{3}}^{x}(x, y, z)+ \\
& \sum_{l_{1}=1}^{r} \sum_{l_{2}=1}^{r+1} \sum_{l_{3}=1}^{r} H_{K, l_{1} l_{2} l_{3}}^{y}(t) \vec{\psi}_{l_{1} l_{2} l_{3}}^{y}(x, y, z)+ \\
& \sum_{l_{1}=1}^{r} \sum_{l_{2}=1}^{r} \sum_{l_{3}=1}^{r+1} H_{K, l_{1} l_{2} l_{3}}^{z}(t) \vec{\psi}_{l_{1} l_{2} l_{3}}^{z}(x, y, z)
\end{aligned}
$$

where

$$
\begin{aligned}
\forall\left(K, l_{1}, l_{2}, l_{3}\right) \in \tau_{h} \times[1, r] \times[1, r+1] \times[1, r+1] \\
\vec{\varphi}_{l_{1} l_{2} l_{3}, K}^{x}(x, y, z)=L_{l_{1}}^{g} L_{l_{2}}^{g l} L_{l_{3}}^{g l} \overrightarrow{e_{x}} \\
\forall\left(K, l_{1}, l_{2}, l_{3}\right) \in \tau_{h} \times[1, r+1] \times[1, r] \times[1, r+1] \\
\vec{\varphi}_{l_{1} l_{2} l_{3}, K}^{y}(x, y, z)=L_{l_{1}}^{g l} L_{l_{2}}^{g} L_{l_{3}}^{g l} \overrightarrow{e_{y}} \\
\forall\left(K, l_{1}, l_{2}, l_{3}\right) \in \tau_{h} \times[1, r+1] \times[1, r+1] \times[1, r] \\
\vec{\varphi}_{l_{1} l_{2} l_{3}, K}^{z}(x, y, z)=L_{l_{1}}^{g l} L_{l_{2}}^{g l} L_{l_{3}}^{g} \overrightarrow{e_{z}} \\
\forall\left(K, l_{1}, l_{2}, l_{3}\right) \in \tau_{h} \times[1, r+1] \times[1, r] \times[1, r] \\
\vec{\psi}_{l_{1} l_{2} l_{3}, K}^{x}(x, y, z)=L_{l_{1}}^{g l} L_{l_{2}}^{g} L_{l_{3}}^{g} \overrightarrow{e_{x}} \\
\forall\left(K, l_{1}, l_{2}, l_{3}\right) \in \tau_{h} \times[1, r] \times[1, r+1] \times[1, r] \\
\vec{\psi}_{l_{1} l_{2} l_{3}, K}^{y}(x, y, z)=L_{l_{1}}^{g} L_{l_{2}}^{g l} L_{l_{3}}^{g} \overrightarrow{e_{y}} \\
\forall\left(K, l_{1}, l_{2}, l_{3}\right) \in \tau_{h} \times[1, r] \times[1, r] \times[1, r+1] \\
\vec{\psi}_{l_{1} l_{2} l_{3}, K}^{z}(x, y, z)=L_{l_{1}}^{g} L_{l_{2}}^{g} L_{l_{3}}^{g l} \overrightarrow{e_{z}}
\end{aligned}
$$

In these definitions, $r$ defines the spatial approximation order, $L_{l_{1}}^{g}(x)$ the Lagrangian polynomial of degree $r$ whose roots are the Gauss points inside $\left[x_{1 K}, x_{2 K}\right]$ and $L_{l_{1}}^{g l}(x)$ the Lagrangian polynomial of degree $r+1$ whose roots are the Gauss-Lobatto points inside $\left[x_{1 K}, x_{2 K}\right]$. The definitions of $L_{l_{2}}^{g}(y), L_{l_{2}}^{g l}(y), L_{l_{3}}^{g}(z)$ and $L_{l_{3}}^{g l}(z)$, are similar by using $\left[y_{1 K}, y_{2 K}\right]$ and $\left[z_{1 K}, z_{2 K}\right]$. In these definitions $K=\left[x_{1 K}, x_{2 K}\right] \times\left[y_{1 K}, y_{2 K}\right] \times\left[z_{1 K}, z_{2 K}\right]$. Figure 7 represents the location of the basis function in the cartesian cell. Figure 8 shows the interest of choosing such a FEM scheme instead of the usual Yee's scheme to model cartesian cavity

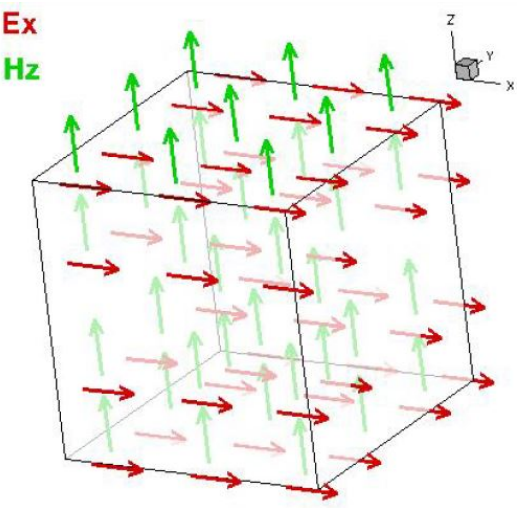

FIGURE 7: FEM method. Location of the electric field and magnetic field basis function for $r=2$ with respect to the $\mathrm{x}$ axis and $\mathrm{z}$ axis respectively.

problems by comparing time-domain result of a $T E_{3,3,0}$ mode inside a $1 \mathrm{~m}$ perfectly metallic cube. The gain obtained in accuracy is obvious.

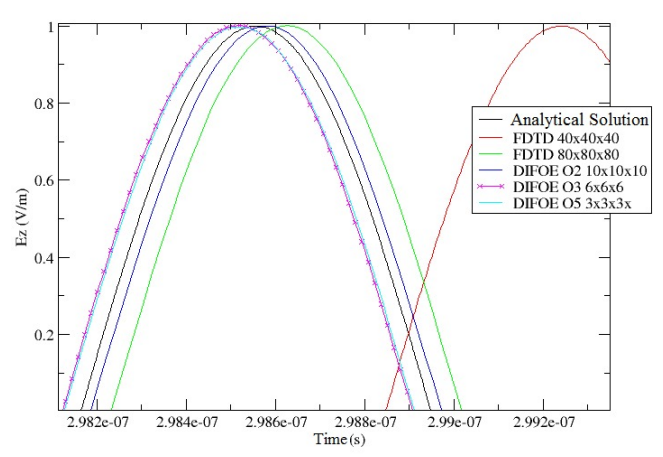

FIGURE 8: $T E_{3,3,0}$ cavity mode inside a $1 \mathrm{~m}$ perfectly metallic cube. Plots labelled "DIFOE" are results obtained with the FEM method with various spatial orders (2, 3 and 5) and various mesh sizes. Plots labelled "FDTD" are results obtained with the Yee's scheme for various mesh sizes.

\section{HYBRIDIZATION TECHNIQUE APPLIED ON A SINGLE-WIRE NETWORK CONFIGURATION}

In the "single-wire" network configuration, we simplify our multiple cavity problem by considering that each branch of the wiring of section II is made of a single wire. The plane wave illumination is the one defined in Figure 1. The connections between the 3 wires are ideal (without losses) at the level of junction $\mathrm{J} 3$ and short-circuit endload conditions are applied at the ends of the 3 wires. The interest of this configuration is to provide the possibility to validate our hybrid technique by comparing it with a full-wave FEM scheme embedding an equivalent Holland thin-wire model [8] of the wiring. For this configuration, 
we will investigate the advantage of having a high spatial approximation order scheme to evaluate the electric fields source terms of the MTLN model and the need to take into account the $J$ current density sources derived from the currents induced on the wires in Maxwell's equations for the update of the EM fields. Figure 9 displays timedomain results obtained with the FEM method of spatial order 1. On the left hand side, we have compared our hybrid method with the reference full-wave approach embedding the thin-wire model. Perfect agreement is obtained between the hybridization and the reference FEM methods. On the right hand side we compare the hybrid method when the electrical current reaction term $J$ is taken or not in the formulation. The results clearly show the importance to take into account in the Maxwell-equations the current-density sources due to the currents induced on the cables. In the classical FTL model, these source terms are not considered : our example demonstrates that this assumption may lead to overestimation of the oscillation amplitudes. Indeed, the hybrid approach implicitly accounts for radiation losses on the wires by its formulation that includes the reaction of the induced currents on the wires on the incident field. Such losses cannot be accounted for in the FTL model.

As far as the FEM approximation order is concerned, the results in Figure 10 present electrical currents in the single-wire configuration for various FEM spatial orders using the full-wave method comparing order 1 and order 2 on the one side and order 2 and order 3 on the other side. The results show the advantage of using a large spatial order approximation scheme in order to obtain an accurate solution without numerical dissipative and dispersive errors. In particular, large spatial orders offer the possibility to use larger mesh cells providing significant gain in memory storage, CPU time and, finally enhancement of the global computational cost. Particularly, Figure 10 shows that a spatial order equal to 2 is sufficient to obtain an accurate solution. This simulation justifies the need to introduce in our hybrid FEM/MTLN technique a high-order scheme to solve Maxwell's equations ; the order 2 will be used for subsequent results in the next MLTN configurations.

\section{HYBRIDIZATION TECHNIQUE APPLIED ON THE MULTICONDUCTOR CONFIGURATION}

After the validation of the field and current exchange process in our hybridization technique, we want now to show the interest of being able to account properly for the multiconductor electrical characteristics of the wiring. To this extent, we are interested in comparing the results obtained on the single-wire configuration with the real multiconductor configuration, both of them being under the plane wave illumination defined in Figure 1. For this, we compared the total currents flowing in each branch of the two network models.

In Figure 11, we show several comparisons of currents obtained on the 3 branches of the network for three configurations :
- the single wire configuration studied in the previous paragraph to validate the hybridization method ;

- the multiconductor configuration with all extremities loaded on short-circuits. This configuration should be the closest to the single wire configuration since, despite the specific connections of the wires, there are short-circuit loads at the extremities ;

- the multiconductor configuration with all extremities loaded on the real end-loads. This configuration preserves both the specific connections between wires as well as the specific loads at the extremities.

In branches 1 and 2, we obtain very similar results in the 3 configurations. This can be explained by the fact that all the cables inside those branches are located in an overshield short-circuited at its both ends. This conductor drives the largest part of the current in the bundles. Branches 1 and 2 are also paths on which the incident electric field, that drives the induced current is maximum. The current being negligible on the cables compared to the overshield, the end loads of those cables do not really matter. From these points of views, the currents on the other cables can be neglected and the single conductor model is appropriate to capture the current induced on the overshield.

On the contrary, in Branch 3, there is no overshield. In the two multiconductor configurations, this branch is made of a shielded pair, which shield is connected to the shield of the coaxial cable in branches 1 and 2, but not to the overshield as mentioned in section II. Since the two wires of the shielded pair are loaded with differential loads at both ends, their induced current is insignificant. Hopefully, the current is much lower on the coaxial-cable shield because the incident field is much lower than in the two other branches, due to the plane-wave illumination configuration. It is then logical that the current induced on this branch is also much lower. However, this is not the case in the single-wire configuration for which the wire in Branch 3 is connected to the two other branches. Such a connection configuration would be quite appropriate only if the shield of the shielded pair was connected to the overshield at the level of $\mathrm{J} 3$, which is not the case as mentioned in section II. Thereby, even if the incident field is low in this branch, the current is linked to the current in branches 1 and 2 by the Kirchhoff law. Note that the current in branch 3 is higher than in the previous configuration and is almost equal to the sum of the currents in Branch 1 and Branch 2.

\section{CONCLUSION}

In this paper, we have presented a hybrid method for coupling Maxwell's equations and MTLN equations in the time-domain for EM-coupling applications of complex multiconductor wiring installed in $3 \mathrm{D}$ scattering objects. This approach presents two main advantages :

- the formulation in the time-domain makes natural at each time-step the reaction of the currents over the fields, which is impossible in the usual FTL approach and which is required at high frequencies when the 

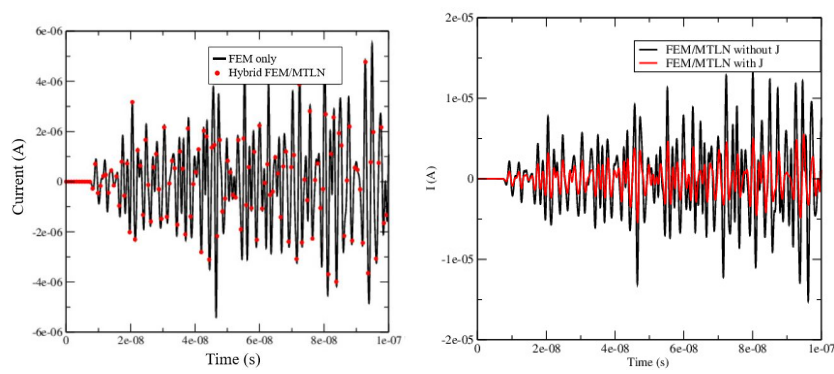

FIGURE 9: Current on wire 1 of tube T1. FEM/MTLN results with and without $J$ current density sources in the Maxwell-equations. "FEM only": full-wave FEM method enbedding the thin-wire model of the wiring. "Hybrid FEM/MTLN": our hybrid method.
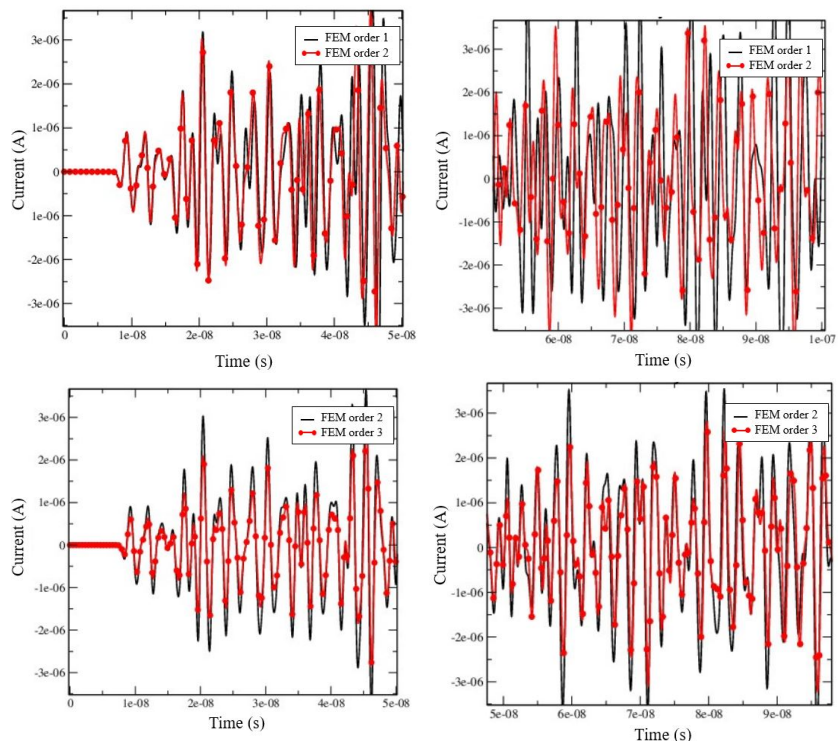

FIGURE 10: Influence of the FEM spatial order on the precision of the solution. Left $[0,5 e-08 s]$, right $[5 e-$ $8 s, 10 e-8 s]$.

TEM mode required by the MTL model is not respected anymore ;

- the MTLN model, in addition to a precise description of the cable bundles provides a realistic description of the multiconductor wiring, which allows us to have all the currents on all the wires.

We have demonstrated these advantages on a generic parallelepiped 3D structure in which a realistic wiring harness made of several types of electric cables was installed. Due to the particular shape of the 3D structure, we have showed the interest of being able to choose the appropriate 3D technique. In our case, our choice fell on a FEM technique dedicated to cartesian meshes and allowing us to obtain high precision on EM fields and gain on the computational cost.

Possible development of this modelling approach may
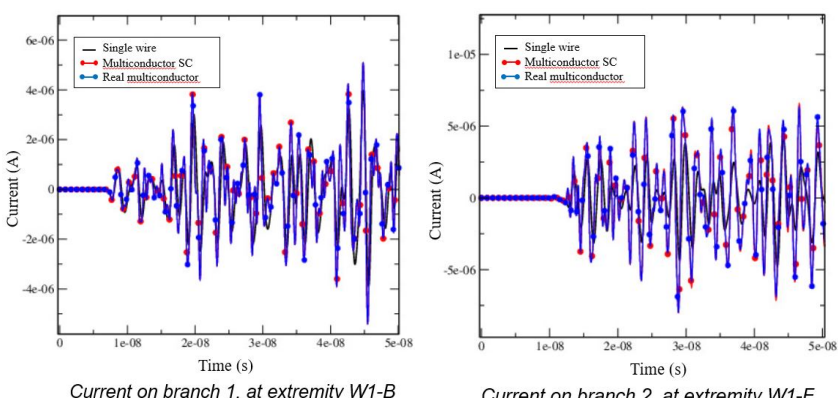

(electric box $A$, at extremity W1-B

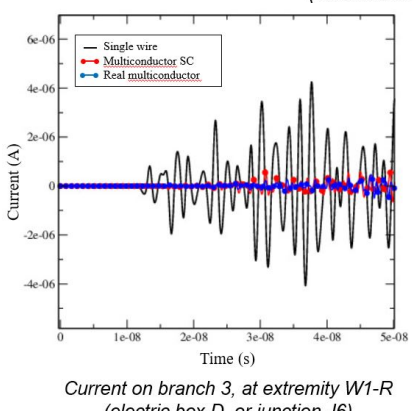

(electric box D, or junction J6)

FIGURE 11: Currents on the network branches. "Single wire": single-wire configuration. "Multiconductor SC": multiconductor configuration with all wires loaded on shortcircuits at their extremities, "Real multiconductor": multiconductor configuration with all wires loaded their real loads at their extremities

concern the investigation of the spatial order required by the MTLN technique with respect to the spatial order chosen for the FEM method.

\section{REFERENCES}

[1] A. Taflove and S. C. Hagness, Computational Electrodynamics: The Finite-Difference Time-Domain Method, Norwood, MA: Artech House (2005).

[2] S.M. Rao Time-Domain Electromagnetics, Series Editors, David J. Irwin Auburn University, Academic Press, 1999.

[3] G.C. Cohen, Higher-Order Numerical Methods for Transient Wave Equations, Scientific Computation, Springer-Verlag, 2002, ISBN 3-540-41598-X.

[4] G.C. Cohen, S. Pernet, Finite Element and Discontinuous Galerkin Methods for Transient Wave Equations, Scientific Computation, Springer-Verlag, 2017, ISBN 978-94-017-7761-2.

[5] R. Holland and L. Simpson, Finite Difference Analysis of EMP Coupling to Thin Structs and Wires, IEEE Trans. on EMC, vol. 23, pp. 88-97, May 1991.

[6] F. Edelvik, A New Technique for Accurate and Stable Modeling of Arbitrary Oriented Thin Wires in the FDTD Method, IEEE Trans. on EMC, 45 (2) : 416423, 2003.

[7] C. Guiffaut, A. Reinex, B. Pecqueux, New Oblique $\square$ 
Thin-Wire Formalism in the FDTD Method With Multi-wire Junctions, IEEE Transactions on Antennas and Propagation, Vol. 60, No. 3, March 2012.

[8] N. Deymier, T. Volpert, X. Ferrieres, V. Mouysset, B. Pecqueux, New High Order FDTD Method to Solve EMC Problem, Advanced Electromagnetics, 4(2), 1725, October 2015.

[9] C. R. Paul, Analysis of Multiconductor TransmissionLines, Wiley-Blackwell (2007).

[10] E. Bachelier, S. Bertuol, J-P. Parmantier, T. Volpert, D. Roissé, N. Muot, C. Giraudon, C. Girard, HIRFSE Cooperative EM Simulation Approach for Modeling the NTC1 Test-case with the ALICE FDTD and CRIPTE Codes, proceedings of CEMEMC'13 workshop. March 19th-21st, 2013

[11] EPICEA: Electromagnetic Platform for lightweight Integration/Installation of electrical systems in Composite Electrical Aircraft. Project Website. Available at http://epicea-env714.eu.

[12] F.M. Tesche, M.V. Ianoz, T. Karlsson, EMC Analysis Methods and Computational Models, John Wiley \& Sons, pp. 247-266, 1997.

[13] A. K. Agrawal, H. J. Price, S. B. Gurbaxani, Transient Response of Multiconductor Transmission-Lines Excited by a Nonuniform Electromagnetic Field, IEEE Transactions on Electromagnetic Compatibility, Vol. EMC-22, No. 2, pp. 119-129, May 1980.

[14] L. Paletta, J-P. Parmantier, F. Issac, P. Dumas, and J.C. Alliot, Susceptibility Analysis of Wiring in a Complex System Combining a 3D Solver and a TransmissionLine Network Simulation, IEEE Trans. on Electromagnetic Compatibility, Vol. 44, No. 2, 309-317, 2002.

[15] X. Ferrieres, J-P. Parmantier, S. Bertuol, and A. Ruddle, Application of Hybrid Finite Difference/Finite Volume to Solve an Automotive Problem, IEEE Trans. on Electromagnetic Compatibility, Vol. 46, No. 4, 624634, 2004.

[16] S. Arianos, M. A. Francavilla, M. Righero, F. Vipiana, P. Savi, S. Bertuol, M. Ridel, J-P. Parmantier, L. Pisu, M. Bozzetti, G. Vecchi, Evaluation of the Modeling of an EM Illumination on an Aircraft Cable Harness, IEEE Transactions on EMC, Vol. 26, 4, Aug. 2014, pp. 844-853

[17] I. Junqua, J-P. Parmantier, S. Bertuol, FTL approach for High Frequency EM Coupling on Cables installed in Complex Structures, In Proceedings of the 2018 International Symposium on Electromagnetic Compatibility-EMC Europe.

[18] J-P. Berenger, A Perfectly Matched Layer for the Absorption of Electromagnetic Waves, J. Comput. Phys. 114, 185-200, 1994.

[19] J-P. Berenger, A Multiwire Formalism for the FDTD Method, IEEE Transactions on EMC, Vol.42, No.3, 257-264, August 2000.

[20] C.E. Baum, T.K. Liu, F.M. Tesche, On the Analysis of General Multiconductor Transmission-Line Networks,
Interaction Notes 350, Air Force Weapons Laboratory, Kirtland Air Force Base, NM, Nov. 1978. Available at http://ece-research.unm.edu/summa/notes/index.html

[21] M. Ridel, P. Savy, J-P. Parmantier, Characterization of Complex Aeronautic Harness - Numerical and Experimental Validations. Electromagnetics, Vol.33, Issue 5, pp. 341-352, 2013.

[22] P. Degauque, J-P. Parmantier, Compatibilité Electromagnétique - Chapitre 2: Couplage aux structures filaires (P. Degauque et J-P. Parmantier). In "Compatibilité Electromagnétique", Collection technique et scientifique des télécommunications. In French.

[23] N. Muot, C. Girard, X. Ferrieres and E. Bachelier, A Combined FDTD/MTL Time-Domain Method to Solve Efficiently Electromagnetic Problems, PIERS B, Vol.58, 409-427, 2013.

[24] C.E. Baum, Low- and High-Frequency Solutions of the Telegrapher Equations for Nonuniform Multiconductor Transmission Lines, Proceedings of the 2007 IEEE International Symposium on Electromagnetic Compatibility, Honolulu, HI, USA.

[25] K.S. Yee, Numerical Solution of Initial Boundary Value Problems Involving Maxwell's Equation in Isotropic Media, IEEE Transactions on Antennas and Propagation, Vol.14,No.3, pp.302-307, May 1966.

[26] J-P. Parmantier, S. Bertuol, and I. Junqua, "CRIPTE : Code de réseaux de lignes de transmission multiconducteur - User's guide-Version 5.1", ONERA/DEMR/T-N119/10 - CRIPTE 5.12010.

[27] R. Chilton, H-, P- and T-Refinement Strategies for the Finite-Difference Time-Domain (FDTD) Method Developped via Finite-Element (FE) Principles, Ph.D. thesis, The Ohio State University, 2008. 